Departments of Endocrinology, Pathology and Surgery at the main children's hospitals.

Results Fifteen cases of gonadoblastoma were identified, all except one phenotypically female. Fourteen patients had prophylactic gonadectomy and one presented with an ovarian mass and raised tumour markers. Eight had TMSY (age at gonadectomy 2 weeks - 14 years). Seven were phenotypically female and one was male. Seven cases of 46 XY GD (all female phenotype) were diagnosed with gonadoblastoma with an age range of 4 months - 15 years at time of surgery. Four of these were unilateral. In the remaining three cases, one patient had bilateral gonadoblastoma, one had unilateral dysgerminoma and contralateral gonadoblastoma and the third had bilateral dysgerminoma with features of gonadoblastoma.

Conclusions This is the first reported population incidence rate of GB in children with a 20 year incidence of gonadoblastoma in the Republic of Ireland of 1/100,000 live births. The data supports the recommendation for elective gonadectomy in high risk conditions. Due to the wide age range in presentations, however, the timing of gonadectomy should be individualised, based on underlying diagnosis and following multidisciplinary team discussion. The true rate of malignant transformation in early onset GB remains to be studied.

\section{OC48 RE-INTERROGATION OF WHOLE EXOME SEQUENCING DATA IN DEVELOPMENTAL EPILEPTIC ENCEPHALOPATHIES}

${ }^{1,2}$ Kathleen Mary Gorman*, ${ }^{2}$ Judith Conroy, ${ }^{1}$ Eva Forman, ${ }^{3}$ Sally A Lynch, ${ }^{4}$ Nicholas M Allen, ${ }^{1}$ Amre Shahwan, ${ }^{1}$ Brian Lynch, ${ }^{2}$ Sean Ennis, ${ }^{1,2}$ Mary King. ${ }^{1}$ Department of Neurology and Clinical Neurophysiology, Temple Street Children's University Hospital, Dublin, Ireland; ' $A$ cademic Centre on Rare Diseases, School of Medicine and Medical Science, University College Dublin, Dublin, Ireland; ${ }^{3}$ Department of Clinical Genetics, Temple Street Children's University Hospital, Dublin, Ireland; ${ }^{4}$ Department of Paediatrics, National University of Ireland, Galway, Galway University Hospital, Galway, Ireland

\subsection{6/archdischild-2019-epa.45}

Introduction The severe epilepsies of infancy and childhood are a heterogeneous group of severe epilepsies characterised by several seizure types, where the epileptic activity in addition to the seizures contributes to cognitive impairment or regression. They account for a significant proportion of the refractory epilepsies and are usually associated with poor outcome. ${ }^{1}$ The tern developmental epileptic encephalopathies (DEE) is now the preferred term for this group of children. It may be the result of a specific congenital or acquired structural brain lesions, metabolic disorders, chromosomal abnormalities, copy number variants or single-gene defects.

Next-generation sequencing (NGS) includes gene panels, whole-exome sequencing (WES) and whole-genome sequencing. The reported rates of diagnosis in DEE using NGS technology ranges from $10-100 \%$. We previously reported a cohort of 50 patients who underwent single research WES for investigations of DEE. The yield at the time of publication was $22 \%$ (11 known epilepsy gene, 1 candidate gene). ${ }^{2}$ 38 patients remained undiagnosed. Since then, the number of new genes reported with DEE continues to expand and the technology improved to aid interpretation of variants. Therefore, we reanalysed WES data, with the addition of parental samples for trio analysis, to enable data interpretation and identification of pathogenic disease-causing variants.
Methods Re-analysis of WES data, single (proband only) or trio (proband and parents)WES, if parental samples were available.

Results We identified a genetic cause in 25 individuals in the cohort; 22 pathogenic variants in DEE genes, 3 candidate genes, increasing the diagnostic yield to $50 \%$. With re-analysis, we identified 10 pathogenic variants (CDKL5, KCNA2, NRXN1, PRODH, RELN, RHOBTB2, SCN1A, SLC1A4, $S M C 1 A), 1$ candidate gene $(N A P B)$ and 1 variant of uncertain significance $(G R I N 2 A)$. A number of genes had not been identified at the time of initial analysis, including RHOBTB2, SLC1A4, SMC1A. Two mosaic variants in CDKL5 and SCN1A were identified with trio WES analysis and reducing read depth filter to 15 , previously set at 20 .

Discussion This study highlights the importance of the reinterrogation of WES data for newly discovered genes. Trio WES had a higher diagnostic yield (50\% compared to 22\%) in keeping with previous studies. Trio WES is effective for the identification of de novo variants and aids in the interpretation of variants. Reducing read depth filter can aid the identification of mosaic variants, increasing reported to be important in DEE.

\section{OC49 NON-ALCOHOLIC FATTY LIVER DISEASE IN OBESE AND OVERWEIGHT IRANIAN CHILDREN: A CROSS SECTIONAL STUDY}

${ }^{1}$ Farzad Maleki, ${ }^{2}$ Marjan Hosseinpour, ${ }^{3}$ Bahman Mansouri motlagh, ${ }^{4}$ Hadi Esmaeili Gouvarchin Ghaleh, ${ }^{2}$ Shahsanam Gheibi*. 'Department of Epidemiology and Biostatistics, School of Public Health, Tehran University of Medical Sciences, Tehran, Iran, Islamic Republic of; ${ }^{2}$ Maternal and Childhood Obesity Research Center, Urmia University of Medical Sciences, Urmia, Iran, Islamic Republic of; ${ }^{3}$ Department of Pathobiology,Faculty Veterinary Medicine, Urmia University, Urmia, Iran, Islamic Republic of; ${ }^{4}$ Applied Virology Research Center Baqiyatallah University of Medical Sciences, Tehran, Iran, Islamic Republic of

\subsection{6/archdischild-2019-epa.46}

Background Non-Alcoholic Fatty Liver Disease (NAFLD), the main cause of childhood liver abnormalities, is rising with the increase of pediatric obesity and overweight. This study aimed to investigate the prevalence of NAFLD and its predisposing factors in children of Urmia province, northwest of Iran.

Methods In this cross-sectional study, 508 overweight and obese children (251 boys) aged 6- 19 years were recruited by convenience sample from the Shahid Motahari Hospital during 2016-2017. The anthropometric and laboratory measurement and abdominal ultrasonography for liver echogenicity and size were conducted. A questionnaire was also used to obtain information on demographical characteristic and alcohol consumption. Eligibility criteria were: (1) $6 \leq$ age $\leq 19$ years; (2) BMI $>85$ th percentile for gender and age (3) absence of any drug toxicity; (5) abstinence from alcohol; (6) absence of hepatitis B, C and Wilson's disease. Fatty liver was diagnosed by ultrasonography using standard criteria. Serum Alanine aminotransferase (ALT), Aspartate aminotransferase (AST), Alkaline Phosphate (ALK), Triglyceride (TG), Cholesterol (Chol), Low density lipoprotein (LDL) -cholesterol, High density lipoprotein (HDL)-cholesterol, Thyroid stimulating hormone (TSH) and glucose, were measured in children blood samples using standard laboratory methods. Insulin resistance was estimated using the homeostasis model assessment of the insulin resistance (HOMA-IR). Univariable and multivariable logistic regression and $95 \%$ confidence interval was used to evaluate predictors of NAFLD. 
Results NAFLD was diagnosed in $8.5 \%$ of overweight and $24.9 \%$ of obese children. In obese children the prevalence of NAFLD was $22.3 \%$ for age group 6-11.9 years and $35.5 \%$ for age group 12-19 years. There was no significant difference between girls and boys $(\mathrm{P}=0.521)$. Age was similar in children with and without NAFLD $(\mathrm{P}=0.766)$. An increase of $10 \mathrm{U} / \mathrm{L}$ of ALT, AST and ALK increased the odds of NAFLD $6 \%, 5 \%$ and $3 \%$, respectively. An increase of $10 \mathrm{mg} / \mathrm{dl}$ of triglycerides and glucose were associated with a $12 \%$ and $8 \%$ increase and one of TSH with a 15\% increase in the odds of NAFLD. An increase of 1 unit of HOMA-IR was associated with a $21 \%$ increase in the odds of NAFLD.

Conclusions ALT, AST, ALK and HOMA-IR can predict the progression of NAFLD. Findings emphasize on the importance of prevention of obesity and early intervention to prevent abnormalities among obese children.

\section{OC50 ACUTE LIVER FAILURE IN CHILDREN: AETIOLOGY AND EVOLUTION}

\footnotetext{
1,2Alina Grama, ${ }^{2}$ Lucia Burac, ${ }^{1,2}$ Simona S Cainap, ${ }^{2}$ Cornel Aldea, ${ }^{2}$ Dan Delean, ${ }^{2}$ Bogdan Bulata, ${ }^{2}$ Diana Pacurar, ${ }^{2}$ Laura Bodea, ${ }^{2}$ Claudia Sirbe, ${ }^{1,2}$ Tudor L Pop*. ${ }^{1}$ University of Medicine and Pharmacy 'Iuliu Hatieganu' Cluj-Napoca, Romania, Cluj-Napoca, Romania; ${ }^{2}$ Emergency Clinical Hospital for Children, Cluj-Napoca, Romania
}

10.1136/archdischild-2019-epa.47

Introduction Acute liver failure (ALF) is a syndrome defined by rapid decline of hepatic function, jaundice, coagulopathy (INR >1.5) and hepatic encephalopathy in patients with no evidence of previous liver disease. The aetiology of ALF in children varies on the age.

Material and methods We have analysed retrospectively the aetiology of the ALF in children followed-up in our clinic during 7-year period (January 2012 - December 2018). We studied the main causes of ALF based on the age of children and their evolution after treatment.

Results During this period, 97 patients (46 males, 47.42\%) were admitted with ALF: 33 infants (0-12 months), 30 children (1-14 years) and 34 adolescents (14-18 years).

In infants the most important causes of ALF were viral and bacterial infections: 24 patients (72.72\%). Cytomegalovirus, Epstein-Barr virus, Herpesvirus, Parvovirus or Gram-negative bacteria were involved, with a high mortality: 10 patients (30.30\%). Metabolic disorders were the cause of ALF in 9 patients (43.47\%): 3 infants with mitochondrial disorder, 2 infants with galactosemia and tyrosinemia respectively, and one with neonatal haemochromatosis and hereditary fructose intolerance. Of those, 3 survived after supportive therapy and diet, one is alive after liver transplantation and 5 died.

In children, toxic hepatitis was the most important cause of ALF (18 patients, 60\%): albendazole in 11 children, mushrooms in 3 children, and one child with each acetaminophen, valproate or fluconazole. Viral and bacterial infections were the causes of ALF in 5 children (16.66\%), 2 of them developed fatal aplastic anaemia. There were also 4 children with autoimmune hepatitis (AIH, 13.33\%) and 3 children with Wilson disease (WD, 10\%) presenting ALF at the time of diagnosis. The mortality in children with ALF was $20 \%$ (6 patients).

Drugs and mushrooms intoxication was the most common cause of ALF among adolescents: 27 patients (79.41\%), acetaminophen being the most often involved (17 patients). Fortunately, the mortality was low and only one patient died after colchicine overdose. There were 4 patients with $\mathrm{AIH}$ and 4 with WD. One patient died with Fulminant WD, other 2 were transplanted and the others are alive with supportive therapy.

Conclusions In children, the aetiology of ALF is different depending on age and is associated with a high mortality despite optimal medical therapy, but without emergency liver transplantation. The management of this cases requires a multidisciplinary team involving paediatric hepatologist, critical care specialist, nephrologist or liver transplant surgeon.

\section{OC51 ANTI-PARIETAL CELL ANTIBODIES IN CHILDREN WITH CELIAC DISEASE. GLUTEN FREE DIET EFFECT}

1,2 Valeria Novicova, ${ }^{1}$ Natalia Shapovalova*, ${ }^{1}$ Maria Revnova, ${ }^{3}$ Elena Kalinina, ${ }^{2}$ Sergei Lapin, ${ }^{1}$ Olga Gurina, ${ }^{1}$ Elena Dementieva, ${ }^{1}$ Ruslan Nasyrov. ${ }^{1}$ St. Petersburg State Pediatric Medical University, St. Petersburg, Russian Federation; ${ }^{2}$ St. Petersburg First Medical University, St. Petersburg, Russian Federation; ${ }^{3}$ Mechnikov I.I. North-Western State Medical, St. Petersburg, Russian Federation

\subsection{6/archdischild-2019-epa.48}

Aim To evaluate the prevalence of anti-parietal cell antibodies (APCA) in children with celiac disease(CD) and gluten free diet (GFD) effect

Methods The first group consisted of 58 children with chronic gastritis (CG) and newly diagnosed CD who didn't adhere to the GFD, the second group included 49 children with CG and CD, who were on GFD. The comparison group 3 consisted of 69 children with CG and excluded CD. To confirm or exclude $\mathrm{CD}$, according to the recommendations of ESPHGAN, the serological examination (anti-tTG IgG, IgA and anti-DPG IgG by ELISA), morphological study, were carry out for all patients. And 80 patients underwent genetic testing to determine the HLA-DQ2/HLA-DQ8 genes using PCR.

All patients underwent a same examination: histological study of gastric biopsies, histological verification of $\mathrm{H}$. pylori infection and biopsy urease test. Identification of APCA: antiIntrinsic Factor (ELISA) was carried out in 140 patient and anti- $\mathrm{H}+/ \mathrm{K}+$ ATPase (ELISA) in 58. APCA, using indirect immunofluorescence reaction, were determined in 45 children. Immunohistochemical determination of anti-tTG deposits in the stomach and duodenal mucous was performed on 10 patients.

Results The frequency of elevated levels of APCA was in group $1-10,00 \%$, in group $2-0 \%$, in group $3-5,43 \%$ $\left(\mathrm{P}_{1,2}=0,012 ; \mathrm{P}_{1,3}=0,492 ; \mathrm{P}_{2,3}=0,065\right)$. The prevalence of fundal gastric atrophy didn't differ statistically significant in groups, $p>0,05$ Correlation analysis didn't reveal morphometric duodenal mucosal parameters association with the gastric mucosa in group 1 and $3, p>0,05$. In group 2 a strong direct correlations between the depth of the fundal gastric pits and depth of the duodenal crypts $(r=0,818)$ and between number of parietal cells and intraepithelial lymphocytes in duodenum $(\mathrm{r}=-0,369) ; \mathrm{p}<0,05$ were found. Deposits of anti-tTG antibodies was detected in duodenal mucous and wasn't found in gastric mucous. APCA didn't correlate with anti-tTG antibodies, $\mathrm{p}>0,05$.

Conclusion The prevalence of APCA in children with CD (1:10) was higher than in children with isolated gastritis (1:20), but statistically not significant. However, the prevalence of APCA is less among patients on a GFD statistically significant. The possible protective effect of a GFD on APCA requires additional study. 